\title{
The effect of intensity and frequency of intermittent punishment in acquisition on resistance to extinction of an approach response in the rat
}

\author{
DAVID R. LINDEN \\ West Liberty State College, West Liberty, West Virginia 26074
}

\begin{abstract}
Rats trained to make an approach response with either nonreward or 150- or $175-\mathrm{V}$ shock occurring on 2,3 , or 4 of 6 daily trials showed greater resistance to extinction than continuously reinforcedunpunished controls. Persistence during extinction was a function of both the type and the frequency of response-contingent event in acquisition. The significant interaction of these two factors was best interpreted as a result of the counterconditioning of the approach response (Amsel, 1972) to anticipatory conditions which varied in similarity to the frustrative nonreward of extinction.
\end{abstract}

While investigations of the transfer of persistence between punishment and frustrative nonreward have been interpreted to indicate a functional similarity between fear ( $\mathrm{rp}-\mathrm{Sp})$ and anticipatory frustration $\left(\mathrm{r}_{\mathrm{F}}-\mathrm{S}_{\mathrm{F}}\right)$ (Brown \& Wagner, 1964; Linden \& Hallgren, 1973), there have been few attempts to manipulate this commonality experimentally, to test Wagner's (1969) statement that frustrative nonreward is less aversive than punishment. Linden (1974a) found that punishment by $150 \mathrm{~V}$ on $50 \%$ of acquisition trials produced greater resistance to extinction than punishment by $175 \mathrm{~V}$. This finding indicated that the lower intensity of shock produced a magnitude of $r_{P}-S_{P}$ that was more similar to the $r_{F}-S_{F}$ of extinction. However, an intensity of shock which did not increase response times during acquisition, $125 \mathrm{~V}$, did not increase resistance to extinction over that of the continuously reinforced-unpunished control animals.

These results are consistent with Amsel's (1972) theory of persistence, as well as with the earlier conditioning-model theory (Amsel, 1958, 1962). The 125-V shock was not a disruptive event, i.e., it did not produce an interfering response, hence counterconditioning of the approach response to sp did not occur. The more intense punishments did have a disruptive effect (produced interfering response), and counterconditioning of the approach response to the $s_{p}$ conditioned on the basis of the $150-$ and $175-\mathrm{V}$ punishment did occur during training; hence they produced greater resistance to extinction.

The purpose of the present investigation was to determine the effect of manipulating both the frequency (percentage) and intensity of punishment during acquisition on resistance to extinction. Since the conditioning model holds that $r_{\mathrm{P}}-\mathrm{s}_{\mathrm{P}}$ as well as
$\mathrm{r}_{\mathrm{F}}-\mathrm{S}_{\mathrm{F}}$ is established by mechanisms of classical conditioning, varying the frequency of punishment will influence the magnitude of $r_{P}$-sp produced by a given shock intensity during training (McAllister \& McAllister, 1971). Therefore, the similarity of $r_{P}-s_{P}$ to the $r_{F}-s_{F}$ of extinction will differ and will increase or decrease resistance to extinction. This interpretation would predict a significant Frequency by Intensity interaction on resistance to extinction.

\section{METHOD}

\section{Subjects}

Eighty-four experimentally naive male Sprague-Dawley rats, $120-150$ days of age, served as subjects. These animals were maintained at $75 \%-80 \%$ of their free-feeding preexperimental weights by a daily ration of $10-15 \mathrm{~g}$ of Wayne Lab Blox given at least $15 \mathrm{~min}$ after each experimental session, water being continuously availabie in the home cages. The experiment was run in four replications of 21 rats each.

\section{Apparatus}

The apparatus was a straight alley with a $91.44 \times 8.25 \times$ $12.70 \mathrm{~cm}$ runway and $30.48 \times 8.25 \times 12.70 \mathrm{~cm}$ start- and goalboxes. The black guillotine doors of the start- and goalboxes were operated by remote control, and the outside of the transparent Plexiglas walls were covered by black construction paper. Noyes pellets $(45 \mathrm{mg}$ ) were placed in a 5.08 -cm-diam, 1.90 -cm-deep glass coaster against the end wall of the goalbox. The floor consisted of aluminum rods placed on $1.27-\mathrm{cm}$ centers. Unscrambled electric shock from a variable voltage dc shock supply was delivered to the floor of the goalbox through $250-\mathrm{K}$-ohm resistance. Shock duration of $.10 \mathrm{sec}$ was controlled by closing one side of the shock circuit through an event timer which was activated by the experimenter via a hand-held push button.

Response times were measured to the nearest $.01 \mathrm{sec}$ by a timer which was activated by the opening of the startbox door and stopped when the subject interrupted an infrared light beam $1.27 \mathrm{~cm}$ in front of the food cup.

\section{Procedure}

Ten days prior to the start of the experiment, the subjects were placed in individual cages and the daily feeding ration was 
initiated. Starting on the 5 th day preceding the start of the experiment, each animal was given a 5-min exploration period in the alleyway. During the last 2 preexperiment days, a $45-\mathrm{mg}$ pellet was placed in the food cup and the subject was removed to his home cage when this pellet was consumed. Each rat received three exploration periods with pellets on these 2 days.

The first 4 days of the experiment consisted of six continuously reinforced trials for all animals. On each trial, the rat was placed in the startbox oriented toward the door which was opened 3-5 sec later. Ten seconds after picking up the $45-\mathrm{mg}$ pellet, the subject was removed to its home cage for a 10-min ITI.

On the 5th experimental day, differential treatment was initiated and continued for 10 daily sessions. The experimental treatment groups consisted of the factorial arrangement of the three responsecontingent events, nonreward $(\mathrm{N})$, shock of $150 \mathrm{~V}$, and shock of $175 \mathrm{~V}$, and four frequencies of occurrence, $0,2,3$, or 4 of the 6 trials of each session. Eight subjects were assigned randomly to each of the nine groups which received an aversive responsecontingent event, and four were assigned to each of the three 0 -frequency groups.

The trials on which nonreward or punishment was to occur were determined randomly prior to each session, with the restriction that the aversive response-contingent event could not occur on four consecutive trials within one session. The rats were retained in the goalbox for $10 \mathrm{sec}$ on both rewarded and nonrewarded trials. On punished trials, shock of $.10 \mathrm{sec}$ duration was delivered .5 to $1.0 \mathrm{sec}$ after the rat picked up the $45-\mathrm{mg}$ pellet; no animal was observed to drop a pellet upon delivery of shock, nor were any pellets found under the grid floor of the goalbox at the end of any session.

Following the experimental treatments, all subjects were administered 10 daily sessions of six nonrewarded trials. Any animal that failed to reach the food cup within $120 \mathrm{sec}$ of the start of a trial was removed to its home cage to await the next trial. A subject that failed to respond for all six trials of a session was terminated from the experiment and assigned a time of $120 \mathrm{sec}$ for the remaining trials.

\section{RESULTS}

Daily median response times were employed in the analysis of the results. Since neither the main effect of replications nor any of its interactions was significant in any of the analyses of variance performed, they have been pooled into the appropriate error term in the analyses reported below. For group comparisons and analysis of simple main effects, the three 0 -frequency groups $(n=4)$ have been pooled to form a continuously reinforced-unpunished control group $(n=12)$, which shall be referred to as group C.

\section{Acquisition}

Figure 1 presents the average daily median response times for the nine experimental groups and the pooled control group. As can be seen in Figure 1, all of the punished groups, except 150-2, showed greater response times than group $\mathrm{C}$ at the initiation of the experimental treatments. All of these groups, except 175-4, appear to have recovered from this suppression by the 4th day of punishment; group 175-4 recovered by the 9 th acquisition day.

A repeated measures analysis of variance, with response-contingent event $(\mathrm{N}, 150 \mathrm{~V}$, or $175 \mathrm{~V})$ and

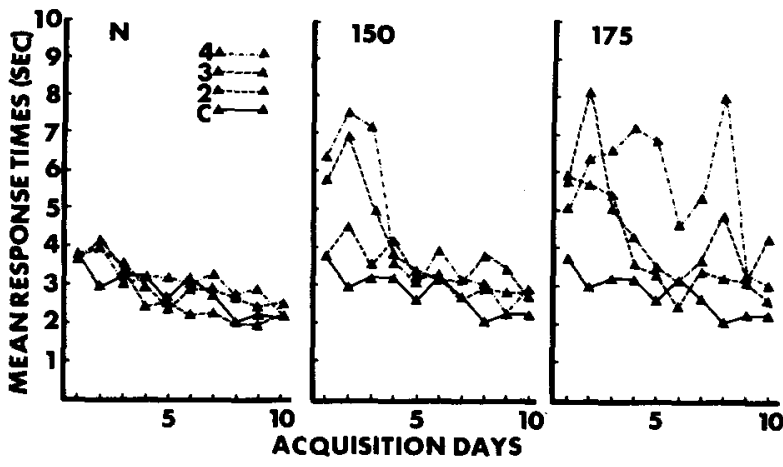

Figure 1. Mean median response times of the groups administered 2,3 , or 4 response-contingent presentations of nonreward, 150-V, and 175-V shock (left-to-right panels) and the continuously reinforced-unpunished group $C$ for the 10 daily acquisition sessions.

its frequency of occurrence $(4,3,2$, or 0$)$ between subjects and halves of training (Days 1-5 vs. 6-10) within subjects, and days within halves, was carried out on the acquisition data (Kirk, 1968; Winer, 1972). Over the 10 days of training, there was no significant effect of either response-contingent event, $F(2,72)=2.98, p>.05$, the frequency of occurrence, $F(3,72)=1.37, p>.10$, or their interaction, $F(6,72)<1.00$. Within subjects, both the effects of halves and days within halves were highly significant, $\mathrm{F}(1,72)=12.82$, and $\mathrm{F}(4,288)=11.24$, ps $<.001$, respectively, while their interaction approached significance, $F(4,288)=2.13, .10>p>.05$. None of the interactions of response-contingent event and frequency of occurrence with halves and/or days was significant (all ps $>.10$ ).

Since the suppressive effects of punishment may have been masked in the overall analysis by both the apparent recovery from these effects and their combination with the nonsuppressive conditions of nonreward and zero frequency, group comparisons and analysis of simple main effects were carried out on these data. Changes in performance during training were assessed by analyzing the simple main effect of days in each half of training for each of the 10 groups. A Newman-Keuls test of the means for these groups for the first and second 5 days of training was also conducted. Finally, one-tailed Dunnett's tests comparing the experimental groups with the continuously reinforced-unpunished control group on each day of the training were carried out.

The control group showed no effect of days in either half of training, $F s(4,288)<1.00$, or a significant change in performance from the first to the second half of training $(p>.05)$. The three nonreward groups (N-2, N-3, N-4) did not differ from the controls or each other in either the first or second half of training ( $p s>.05$ ), nor did any of these groups show an effect of days in either half of training, $F s(4,288)<1.00$. As in earlier reports, the 
nonreward conditions had no suppressive effect on performance of the approach responses (Linden, 1974a, b).

Group 150-2 showed no significant difference from the nonpunished control and nonreward groups (ps $>.05$ ). Nor did they show a significant change from the first to the second half of training $(p>.05)$ or a significant effect of days in either half, Fs $(4,288)<1.00$. However, it is evident that this condition did have a mild suppressive effect. As a whole, group 150-2 showed slight increases in response times on Training Days 2 and 4 (see Figure 1), and six of the eight animals in this group demonstrated reliable increases in response times, at least doubling the preceding day's time, but these increases were not consistent among animals.

This inconsistency of suppression was also evident in group 175-2, which did not differ from the control group on any single day (all Dunnett ps $>.05$ ) but did differ from the control and nonreward groups over the first 5 days (ps $<.05$ ). Furthermore, this group showed a significant reduction in response time from the first to the second half of training $(p<.05)$ and did not differ from the nonpunished groups during the second half ( $p s>.05$ ). The effect of days only approached significance during the first 5 days of training, $F(4,288)=1.97, p=.10$, and was completely absent during the second 5 , $F(4,288)<1.00$. Thus, it can be concluded that the two-punishment-per-day conditions did produce a mild suppressive effect from which recovery was fairly rapid. Hence, counterconditioning of the approach response had presumably occurred.

Suppression and recovery were more pronounced in groups 150-3, 150-4, and 175-3. These groups all differed from the nonpunished groups over the first 5 days of training ( $<<.05$ for 150-3; $p<.01$ for 150-4 and 175-3). All three had significantly longer response times than group C on Day 2 (Dunnett ps $<.05$ for $150-3$ and $150-4$, and $<.01$ for $175-3$ ), and 150-4 continued to differ from the controls on Day $3(p<.05)$. The recovery of the response over the first 5 days of training was indicated by a significant effect of days for this half, Fs $(4,288)=2.19$, $589,4.41$; ps <.05, < .001, <.005 (for 150-3, 150-4, and $175-3$, respectively), and by the significant change in performance from the first to the second half for all three groups (all ps $<.05$ ). These three groups did not differ from the nonpunished groups during the second half of training ( $\mathrm{ps}>.05$ ), nor was the effect of days significant during this period, Fs $(4,288)<1.00$. Thus it can be concluded that these three groups showed more reliable suppression of the approach response than the two punishment conditions, and complete recovery of that response to the level of the unpunished controls prior to the second five training sessions. Again, countercondi- tioning of the approach response had presumably occurred for these three groups.

Group $175-4$, on the other hand, demonstrated suppression for more sessions and less recovery of the approach response than the other punished groups. Group 175-4 differed significantly from the nonpunished groups over the first and second 5 days of training (ps $<.01$ ), and it showed significantly longer response times than did group $\mathrm{C}$ on Days 2, 4, 5, and 8 (Dunnett ps $<.05,<.05,<.05,<.01$, respectively). Furthermore, there was no effect of days for this group during the first half of training, $F(4,288)<1.00$. However, this group did show a reduction in response times during the second 5 days of training, which was indicated by a significant effect of days for that period, $F(4,288)=5.27$, $\mathrm{p}<.001$, and a significant difference in its performance between the first and second half $(p<.05)$. Thus, recovery of the approach response did occur in group 175-4, but it was not as complete, or for as many sessions, as in the other punished groups (175-4 did not differ from C on Days 9 and 10). Whether this shorter period of recovery was sufficient counterconditioning to increase persistence of the approach response can only be determined from the extinction data.

The suppression during punishment data do not correspond exactly to the expected differences in the magnitude of $\mathrm{r}_{\mathrm{P}} \mathrm{-s} \mathrm{P}$. From the literature on classical fear conditioning, it was expected that increasing either the frequency or intensity of shock should increase the level of conditioned fear (McAllister \& McAllister, 1971). However, the only significant differences among the punished groups were that during the first 5 days of training group 150-2 showed less suppression than the other groups (ps $\lessdot .05$ ) and during the second 5 days group 175-4 was more suppressed, or slower to recover, than the other groups (ps $<.05)$. These results indicate that the alleyway procedure may not be sensitive as a measure of conditioned fear, since suppression and recovery of responding may be determined by factors other than the level of fear produced by the moderate intensities of punishment employed here (D'Amato, 1969). Nevertheless, within each shock intensity, the number of sessions during which significant suppression occurred was a direct function of the frequency of punishment.

\section{Extinction}

The mean median response times for the 10 days of extinction are presented in Figure 2, which shows that the three aversive response-contingent events produced increased resistance to extinction at the three frequencies employed. But the optimal frequency for increasing resistance to extinction differed for the three response-contingent events. 


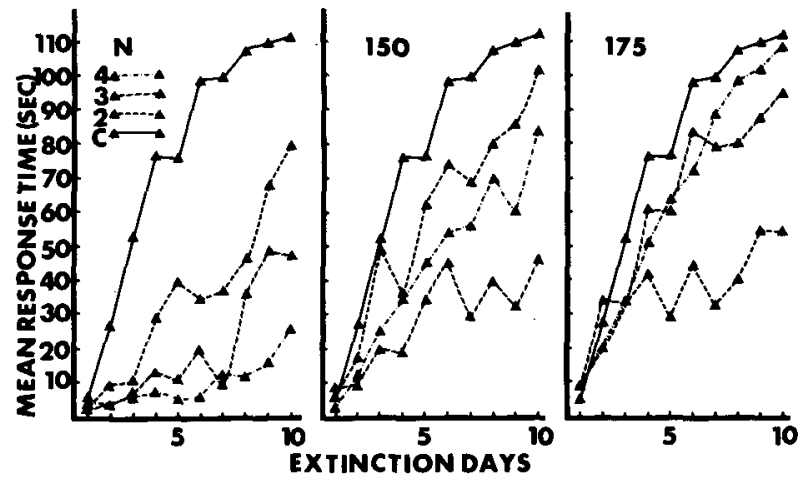

Figure 2. Mean median response times for the 10 days of extinction for the groups administered 2,3 , or 4 presentations of nonreward, 150-V, and 175-V shock (left-to-right panels) and continuously reinforced without punishment (group C) during acquisition.

Furthermore, there appears to be a considerable degree of interaction between the events and their frequency of occurrence.

A repeated measures analysis of variance, with response-contingent event and frequency of occurrence during acquisition between subjects and days of extinction within subjects (Winer, 1972, Case II), indicated a highly significant effect of both responsecontingent event, $\mathrm{F}(2,72)=14.61, \mathrm{p}<.001$, and the frequency of occurrence, $F(3,72)=12.09, p<.001$, as well as their interaction, $F(6,72)=4.98, p<.001$. Within subjects, the effect of extinction days was significant, $F(9,648)=77.75, \mathrm{p}<.001$, as was its interaction with response-contingent event, $\mathrm{F}(18,648)=2.06, \mathrm{p}<.01$, and frequency of occurrence, $F(27,648)=2.49, p<.01$, and three-way interaction, $F(54,648)=1.82, \mathrm{p}<.01$.

In order to assess the differences among the 10 groups, a Newman-Keuls test was conducted. All of the groups that had been administered an aversive response-contingent event, nonreward, $150 \mathrm{~V}$, or $175 \mathrm{~V}$, were significantly more resistant to extinction than the continuously reinforced-unpunished control group (ps $<.01$ ). The three nonreward groups, N-4, N-3, and N-2, ranked first, second, and fourth, respectively, among the 10 groups and differed significantly from each other (ps $<.01$ ). The 150-V groups showed a curvilinear relation, ranking sixth, third, and seventh, 150-4, 150-3, $150-2$, respectively, and differed significantly from each other (ps $<.01$ ). The $175 \mathrm{~V}$ groups showed a reversal, ranking ninth, eighth, and fifth, 175-4, $175-3,175-2$, respectively, among the 10 groups, with groups 175-4 and 175-3 not differing $(p>.10)$ and both differing significantly from $175-2$ (ps $<.01$ ). Thus, for each response-contingent event, a different frequency of occurrence was optimal for producing greater resistance to extinction.

The Newman-Keuls comparisons of groups receiving different response-contingent events during acquisition indicated a high degree of overlap among the nonreward, 150-V and 175-V groups. Group 150-3, ranked third among the 10 groups, was significantly less resistant to extinction than Groups $\mathrm{N}-4$ and $\mathrm{N}-3$, ranked first and second $(p<.01)$, but more resistant than group $\mathrm{N}-2$, ranked fourth $(\mathrm{p}<.05)$. Group 175-2, ranked fifth, did not differ from Group $\mathrm{N}-2$ $(p>.10)$, and both of these groups were more resistant than 150-4, the sixth group $(p<.05)$. Group 150-4, in turn, was significantly different from the seventh, eighth, and ninth groups, 150-2, $175-3,175-4$ (ps $<.01$ ), which did not differ from each other $(p s>.10)$. It can therefore be seen that resistance to extinction as a function of the frequency of occurrence of the response-contingent event during training was a direct relation for nonreward, curvilinear for $150 \mathrm{~V}$ and inverse for $175 \mathrm{~V}$. Hence, resistance to extinction was a function of the intensity and frequency of the aversive responsecontingent event to which the subject was exposed during training.

\section{DISCUSSION}

The present results are consistent with and extend previous reports of increased resistance to extinction following training in which continuous reward is accompanied by intermittent punishment (Brown \& Wagner, 1964; Linden, 1974a), and they support Amsel's (1972) general theory of persistence. All of the animals which were exposed to either intermittent punishment or partial reward during training demonstrated greater resistance to extinction than the continuously reinforced-unpunished control group. Furthermore, the punished groups showed different degrees of response suppression and recovery during training.

While all of the punished groups did show behavioral habituation or counterconditioning of the approach response to $\mathrm{r}_{\mathrm{P}-\mathrm{S}_{\mathrm{P}}}$ (Amsel, 1972), the magnitude of the $r_{P}-s_{P}$ to which this counterconditioning occurred appears to be an important factor in the transfer of persistence. This is evident from the significant difference in resistance to extinction demonstrated by groups which showed very little or no difference in suppression and recovery during training, i.e., $150-2$ vs. $175-2$ and $150-3$ vs. $175-3$. Since these groups did not differ in number of sessions during which counterconditioning was evident, the difference in their performance during extinction must be attributed to the difference in the magnitude of $\mathrm{r}_{\mathrm{P}}$-Sp present during training. In both of the above comparisons, the $175-\mathrm{V}$ conditions would produce greater conditioned fear (McAllister \& McAllister, 1971). Thus it may be concluded that the $r_{F}-s_{F}$ that was produced during extinction was 
more similar to the $\mathrm{r}_{\mathrm{P}}$-sp generated by $150 \mathrm{~V}$ on three of six trials $(50 \%)$ than the other punishment conditions employed in this investigation.

This interpretation implies that in partial reward schedules, lower densities of reward should generate an $\mathrm{r}_{\mathrm{F}}-\mathrm{S}_{\mathrm{F}}$, to which the approach response is counterconditioned in acquisition, that is more similar to the $\mathrm{r}_{\mathrm{F}}-\mathrm{s}_{\mathrm{F}}$ of the $100 \%$ nonreward of extinction. This hypothesis is consistent with the general finding that lower densities of reward, that can maintain responding, produce greater resistance to extinction (Robbins, 1971).

One difficulty with this interpretation is that the $\mathrm{r}_{\mathrm{F}}-\mathrm{S}_{\mathrm{F}}$ produced by the continuous nonreward of extinction seems to be more similar to the $\mathrm{r}_{\mathrm{P}}-\mathrm{s}_{\mathrm{P}}$ of moderate punishment, such as $150-3$, than that of milder punishment, such as $150-2$, or the $r_{F}-S_{F}$ of denser partial reward schedules, such as $\mathrm{N}-2$. However, Wagner (1969) has indicated that frustrative nonreward is less aversive than punishment. It appears that the magnitude of $R_{F}$ and hence $r_{F}-s_{F}$ of extinction is smaller following partial reward than following continuous reward. Spence's (1960) statement that incentive motivation $(K)$, a determinant of $\mathbf{R}_{\mathbf{F}}$, is smaller with partial than with continuous reward is in line with this possibility. Furthermore, exposure to a stimulus situation, such as nonreward, reduces the magnitude of the unconditioned response to that stimulus, but this habituation does not generalize to similar stimuli (Gormezano \& Moore, 1969; Hilgard \& Bower, 1966). Hence, it is possible that the $R_{F}$ and $r_{F}-S_{F}$ of extinction following continuous reward is more aversive than that following partial reward and is thus more similar to the $\mathbf{R}_{\mathbf{P}}$ and $\mathrm{r}_{\mathrm{P}}$-sp produced by intermittent punishment.

While the present investigation was not designed to test explicitly a sequential analysis (Capaldi, 1967; Capaldi \& Levy, 1972), the results of the intermittent punishment treatments do not seem to conform to the predictions of this position. Although the restriction that nonreward or punishment could not occur on more than three consecutive trials limited the maximum $\mathbf{N}$ and $\mathbf{P}$ lengths, it is obvious that the schedules with the greater percentages of nonreward or punishment trials should contain more $\mathbf{N}-\mathbf{R}$ or $P-R$ transitions, or longer $N$ or $P$ lengths, which should produce greater resistance to extinction. However, in the punishment conditions, those subjects that received the most punishments were not the most resistant to extinction.

Nor are the present results congruent with a form of the learned helplessness hypothesis proposed by Rosellini and Seligman (1975). This position, following a suggestion of Amsel, proposes that the increased resistance to extinction following experience with shock may be a form of learned helplessness in which the animal fails to stop running into frustration.
Although the parameters for establishing helplessness in rats have not been specified (Seligman \& Beagley, 1975), it would seem that animals receiving the most frequent and intense shocks during training (175-4) should have developed a level of helplessness equal to or greater than any of the other experimental groups, and should therefore have shown maximum resistance to extinction. The present results do not support such a prediction. Rather, group 175-4 was significantly less persistent than groups exposed to less intense and/or less frequent shocks.

The present results are therefore best interpreted in terms of Amsel's (1972) general theory of persistence and Wagner's (1969) hypothesized similarity of punishment and frustrative nonreward. They can be taken to mean that resistance to extinction following continuous reward accompanied by intermittent punishment was a function of the similarity of $\mathrm{r}_{\mathrm{F}}-\mathrm{S}_{\mathrm{F}}$ in extinction to the $\mathrm{r}_{\mathrm{P}}-\mathrm{S}_{\mathrm{P}}$ to which the approach response was counterconditioned during acquisition. The magnitude of $r_{P}-s_{P}$ conditioned during acquisition is determined by both the frequency and intensity of the punishing shock.

\section{REFERENCES}

AMSEL, A. The role of frustrative nonreward in noncontinuous reward situations. Psychological Bulletin, 1958, 55, 102-119.

AMSEL, A. Frustrative nonreward in partial reinforcement and discrimination learning: Some recent history and a theoretical note. Psychological Review, 1962, 69, 306-328.

AMSEL, A. Behavioral habituation, counterconditioning, and a general theory of persistence. In A. H. Black \& W. F. Prokasy (Eds.), Classical conditioning II: Current research and theory. New York: Appleton-Century-Crofts, 1972. Pp. 409-426.

Brown, R. T., \& WAGNER, A. R. Resistance to punishment and extinction following training with shock and nonreinforcement. Journal of Experimental Psychology, 1964, 68, 503-507.

CaPaldi, E. J. A sequential hypothesis of instrumental learning. In K. W. Spence \& J. T. Spence (Eds.), The psychology of learming and motivation: Advance in theory and research (Vol. 1). New York: Academic Press, 1967. Pp. 67-156.

Capaldi, E. J., \& LEVY, K. J. Stimulus control of punished reactions: Sequences of punishment trials and magnitude of reinforcement trials. Learning and Motivation, 1972, 3, 1-19.

D'AMato, M. R. Instrumental conditioning. In M. H. Marx (Ed.), Leaming: Processes. Toronto: MacMillan, 1969. Pp. 35-118.

Gormezako, I. \& More, J. W. Classical conditioning. In M. H. Marx (Ed.), Learning: Processes. Toronto: MacMillan, 1969. Pp. 121-203.

HrLGARD, E. R., \& Bow ER, G. H. Theories of leaming. New York: Appleton-Century-Crofts, 1966.

KIRK, R. E. Experimental design: Procedures for the behavional sciences. Belmont, Calif: Brooks/Cole, 1968.

LINDEN, D. R. The effect of intensity of intermittent punishment in acquisition on resistance to extinction of an approach response. Animal Learning \& Behavior, 1974, 2, 9-12. (a)

LiNDEN, D. R. Transfer of approach responding between punishment, frustrative nonreward, and the combination of punishment and nonreward. Learning and Motivation, 1974, 5, 498-510. (b)

LINDEN, D. R., \& HALlgReN, S. O. Transfer of approach responding between punishment and frustrative nonreward sustained 
through continuous reinforcement. Leaming and Motivation, 1973, 4, 207-217.

McAlisster, W. R., \& MCAllister, D. E. Behavioral measurement of conditioned fear. In F. R. Brush (Ed.), Aversive conditioning and learning. New York: Academic Press, 1971. Pp. 105-179.

Robbins, D. Partial reinforcement: A selective review of the alleyway literature since 1960. Psychological Bulletin, 1971, 76, 415-431.

Rosellin, R. A., \& Seligman, M. E. P. Frustration and learned helplessness. Joumal of Experimental Psychology: Animal Behavior Processes, 1975, 104, 149-157.

Seligman, M. E. P., \& Beagley, G. Learned helplessness in the rat. Journal of Comparative and Physiological Psychology, 1975, 88, 534-541.

SPEnCE, K. W. Behavior theory and learning. Englewood Cliffs: Prentice-Hall, 1960.

W AGNER, A. R., Frustrative nonreward: A variety of punishment. In B. A. Campbell \& R. M. Church (Eds.), Punishment and aversive behavior. New York: Appleton-Century-Crofts, 1969. Pp. 157-218.

WINER, B. J. Statistical principles in experimental design (2nd ed.). New York: McGraw-Hill, 1972. Pp. 539-559.

(Received for publication February 12, 1976; revision received July 26,1976 .) 